\title{
28 Research Square \\ 4 Short Hairpin RNA Lentiviral Vector-Based System for TRIM5a Knockdown in Cynomolgus Monkey Embryonic Fibroblast Cells Against HIV Infection
}

\section{Ruirui MA}

South China Agricultural University

Shihua Yang ( $\nabla$ yangsh@scau.edu.cn )

South China Agricultural University

\section{Research Article}

Keywords: HIV, TRIM5a, RNA interference, Pseudo-virus

Posted Date: February 22nd, 2022

DOl: https://doi.org/10.21203/rs.3.rs-1264721/v1

License: @ (1) This work is licensed under a Creative Commons Attribution 4.0 International License. Read Full License 


\section{Abstract}

Background: The restriction factor, TRIM5a is known to limit retroviral infection at an early post-entry stage in a species-specific manner. The current study was designed to assess the efficacy of TRIM5a in restricting the human immunodeficiency virus (HIV) using a pseudo-viral infection model in cynomolgus monkey embryonic fibroblast cells (CMEFs down the TRIM5a expression using the lentivirus short hairpin RNA (shRNA) knockdown vector system.

Methods: Three shRNAs against the TRIM5a gene were designed and cloned into lentiviral vectors expressing the green fluorescent protein (GFP) and red fluorescent protein (RFP) reporter genes. The stable knockdown of TRIM5a gene expression was confirmed by both real-time RT-PCR as well as Western blot. The lentiviral inducible shRNA vector was then transduced into the CMEFs infected with HIV Pseudo-virus.

Results: We successfully designed and cloned three shRNAs targets into lentiviral vectors to knock down the TRIM5a gene expression. Further, transduction of the shRNAs into the pseudo-viral HIV infected CMEFs showed greater susceptibility to the infection.

Conclusion: Here, for the first time, we successfully knocked down the TRIM5a gene using RNA interference (RNAi) technology and found TRIM5a-null CMEF cells to be susceptible to HIV pseudo-virus infection. Our research provides an important strategy for the establishment of shRNA based lentiviral vector system for gene knockdown in the cynomolgus monkey animal model against HIV infection.

\section{Introduction}

The global HIV/Acquired immunodeficiency syndrome (AIDS) epidemic continues to present a large-scale humanitarian crisis, especially to the world's most vulnerable communities. According to WHO estimates, there are about 36.7 million people infected with HIV the world over ${ }^{1}$. There have been considerable advances in antiretroviral drugs for the treatments that confer near-normal life expectancy to those adherents to these lifelong drug treatment regimens ${ }^{2}$. However, implications of drug toxicity and resistance and viral latency and also addressing the economic and social hurdles are the potential issues dealt by HIV-infected patients. Therefore, it is crucial to develop a more sustained virological remission in HIV-infected patients.

There are reports stating that TRIM5a is a restriction factor that limits retroviral infection at an early post-entry stage in a species-specific manner ${ }^{3}$. Human TRIM5a (huTRIM5a) protein has limited potential to control HIV-1 and other retroviral infections such as the N-tropic murine leukemia virus (N-MLV) and also the HIV-2 infections. Rhesus monkey TRIM5a (rmTRIM5a) and chimeric TRIM5-Cyclophilin A (TRIM5-CypA) fusion protein were found to successfully inhibit primate lentiviruses ${ }^{4-6}$. In some preclinical studies, humanized mouse models transduced with recombinant lentiviral vectors with hybrid TRIM5a isoforms could restrict HIV-1 infection successfully. Notably, engineering HIV-1 resistance without the use of vectors carry a risk of immunogenicity. Therefore, insertional mutagenesis adds to the advantage ${ }^{7,8}$.

Several studies have reported that there are a variety of innate responses in mammalian cells to control retroviral infection, such as receptor variation (1) or blockade (2), activation of enzymes that are responsible for degradation of retroviral nucleic acids (3), and induction of a class of so-called restriction factors that block one 
or more infection pathways such as post-entry or the pre-integration processes during the viral life cycle (4). Therefore, to infect a cell, a retrovirus must either sidestep the defense mechanisms or encode activities to overcome them (5).

RNA interference (RNAi) is one of the most important technological breakthroughs in modern biology. The mechanism involves the delivery of double-stranded RNAs of 21-23nt long oligonucleotides such as the short interfering RNA (siRNA) that activate gene knockdown by binding to the target gene mRNA sequence and disrupt it. Recently, this technique was widely used for gene knockdown in mammalian cells and in several in vivo experiments ${ }^{9}$. Synthetic siRNAs have been successfully delivered into mammalian cells, primary cell lines, and embryonic stem cells, consistently resulted in more than $80 \%$ down-regulation of the target gene expression in all cases ${ }^{10}$. This approach gives a transient effect where mRNA levels of the suppressed target gene in proliferating cells return to normal state by 3-7 days. To suppress the target gene expression for the long-term, alternative approaches have been developed, such as utilizing expression vectors to deliver siRNAs that are stably expressed as short hairpin RNA (shRNA) ${ }^{11}$.

Although small-animal models such as mice, rabbits, and cats have advantages of high reproductive rates and low maintenance costs, these species are distantly related to humans. HIV-1, being highly specific to humans, faces multiple barriers to replicate in these small animals; therefore, using them as HIV models is a hard choice. On the other hand, the Cynomolgus monkey (Macaca fascicularis) is the best suitable animal model for clinical trials and scientific research. Cynomolgus monkey, also known as long-tailed macaque, is widely distributed in Southeast Asia. Although these animals have so far not been used widely in HIV/AIDS research as compared to rhesus-or pig-tailed macaques, recently, the Cynomolgus monkeys have been used increasingly for studies related to the production of Simian immunodeficiency virus (SIV) and HIV vaccines due to the limited availability of Indian rhesus macaques following the export ban in April $1992^{12}$.

The current study was designed to assess the efficacy of TRIM5a in restricting HIV pseudo infection. For this purpose, we knocked down the TRIM5a gene using short hairpin RNA (shRNA), transduced to CMEF cells that were infected by HIV pseudo-virus.

\section{Material And Methods 2.1. Cell culture}

Human embryonic kidney 293T cell lines (CRL-1573, lot number 7681666) obtained from American Tissue Culture Collection (ATCC, Manassas, VA, USA) were cultured in Dulbecco's modified Eagle's medium (DMEM; Gibco, Grand Island, NY, USA) supplemented with 10\% fetal bovine serum (FBS; Gibco), 200 mmol/L glutamine, $10 \mathrm{mM}$ non-essential amino acids, $100 \mathrm{mM}$ sodium pyruvate, 100 units $/ \mathrm{mL}$ penicillin and $100 \mathrm{mg} / \mathrm{mL}$ streptomycin (Sigma-Aldrich, St. Louis, MO, USA) and incubated with $5 \% \mathrm{CO}_{2}$ at $37^{\circ} \mathrm{C}$. The adherent CMEF cells (established and characterized in our laboratory) were grown in T25 cell culture flasks in high-glucose DMEM medium (DMEM; Gibco, Grand Island, NY, USA) supplemented with 10\% fetal bovine serum (FBS; Gibco), 1\% penicillin, and incubated in the same settings as 293T cells. Confluent cells were passaged by trypsin digestion and directly divided onto fresh culture vessels. The growth of cells was monitored every day using an inverted microscope. 


\subsection{Screening TRIM5a target sites, shRNA synthesis, and preparation of lentivirus vector}

The coding region of the cynomolgus monkey TRIM5a gene was obtained from the NCBI GenBank (https://www.ncbi.nlm.nih.gov/nucleotide) and tested for similarity by performing a BLAST to eliminate potential target sequences to match off-target gene of the same species. The potential single-nucleotide polymorphisms (SNP) were eliminated (http://www.ncbi.nlm.nih.gov/projects/SNP/). Three shRNA targeting different regions of the TRIM5a (shTRIM5a) gene were then selected (Fig. 1. A). The shTRIM5a and nontargeting control shRNA (Table 1) and PCR oligos (Table 2) were synthesized by Dalian Bao Biological Co., Ltd. and purified by PAGE.

Table 1

siRNA target sequence

\begin{tabular}{|ll|}
\hline Name & Sequence $\left(5^{\prime}\right.$-3') $^{\prime}$ \\
\hline Sh1 & ATGAAAATTATCAACCTAAATAT \\
Sh2 & CTGTCTCATTCTTCAATATCACA \\
Sh3 & AGCCTGTATTTCCATATTTAAAT \\
\hline
\end{tabular}

Table 2

shRNA primer sequence

\begin{tabular}{|ll|}
\hline Name & Sequence $\left(\mathbf{5}^{\prime}\right.$-3') \\
\hline Sh1-F & GAAAAAAAGCCTGTATTTCCATATTTAAATTGACAGGAAGATTTAAATATGGAAATACAGGCTCA \\
Sh1-R & TCGAGAAAAAAAGCCTGTATTTCCATATTTAAATTGACAGGAAGATTTAAATATGGAAATACAGGCTCA \\
Sh2-F & TGCTGTCTCATTCAATATCACACTTCCTGTCATGTGATATTGAAGAATGAGACAGTTTTTTC \\
Sh2-R & TCGAGAAAAAACTGTCTCATTCTTCAATATCACATGACAGGAAGTGTGATATTGAAGAATGAGACAGCA \\
Sh3-F & TGATGAAAATTATCAACCTAAATATCTTCCTGTCAATATTTAGGTTGATAATTTTCATTTTTTTC \\
Sh3-R & GAAAAAAATGAAAATTATCAACCTAAATATTGACAGGAAGATATTTAAGGTTGATAATTTTTCATCA \\
\hline
\end{tabular}

The lentiviral vector plasmid pLL3.7 was double-digested with EcoRI and BamHI, the linearized vector fragment was recovered, and the double-stranded oligos were annealed transformed into DH5a competent cells, vector extracted using Qiagen miniprep plasmid isolation kit and sequenced at Shanghai Yingke Jieke Company.

\subsection{Synthesis of recombinant shTRIM5a lentivirus vector}

The recombinant shTRIM5a lentivirus vector (LV-CHPF-shRNA 1, 2, and 3) and the non-targeted control lentiviral vector (LV-NC) were prepared and transfected according to the manufacturer's instructions. In brief, the recombinant lentiviral construct was prepared and was transfected to the 293T cells when they were $70-80 \%$ confluent. The vectors were then harvested at time intervals of 24,48 , and $72 \mathrm{~h}$ after transfection.

\subsection{Validation of shTRIMa knockdown in CMEF cell lines}


The CMEF cells were cultured until 70-80\% confluence, and these confluent cells were transfected with recombinant shTRIMa lentiviral vectors in the same way as was performed with 293T cells. The GFP-positive transfected cells were analyzed and sorted by flow cytometry (Canto II, BD, USA) using GFP tag with FL1 channel in a cell sorter (Aria II, BD, USA).

The membrane fusion of $293 \mathrm{~T}$ cells due to lentivirus transfection was confirmed by vesicular stomatitis virus $\mathrm{G}$ (VSV G) glycoprotein that induces cell fusion using a fluorescence microscope.

\subsection{Quantitative real-time RT-PCR (qRT-PCR)}

Total RNA was extracted from CMEF cells using the TRIzol reagent (Invitrogen, Carlsbad, CA, USA). The cDNA was synthesized from the RNA by reverse transcriptase enzyme from the cDNA Synthesis kit. The PCR amplification was performed as per the manufacturer's recommendations. The sequences of primers used for setting the reaction are listed in Table 3.

Table 3

qPCR primer sequence

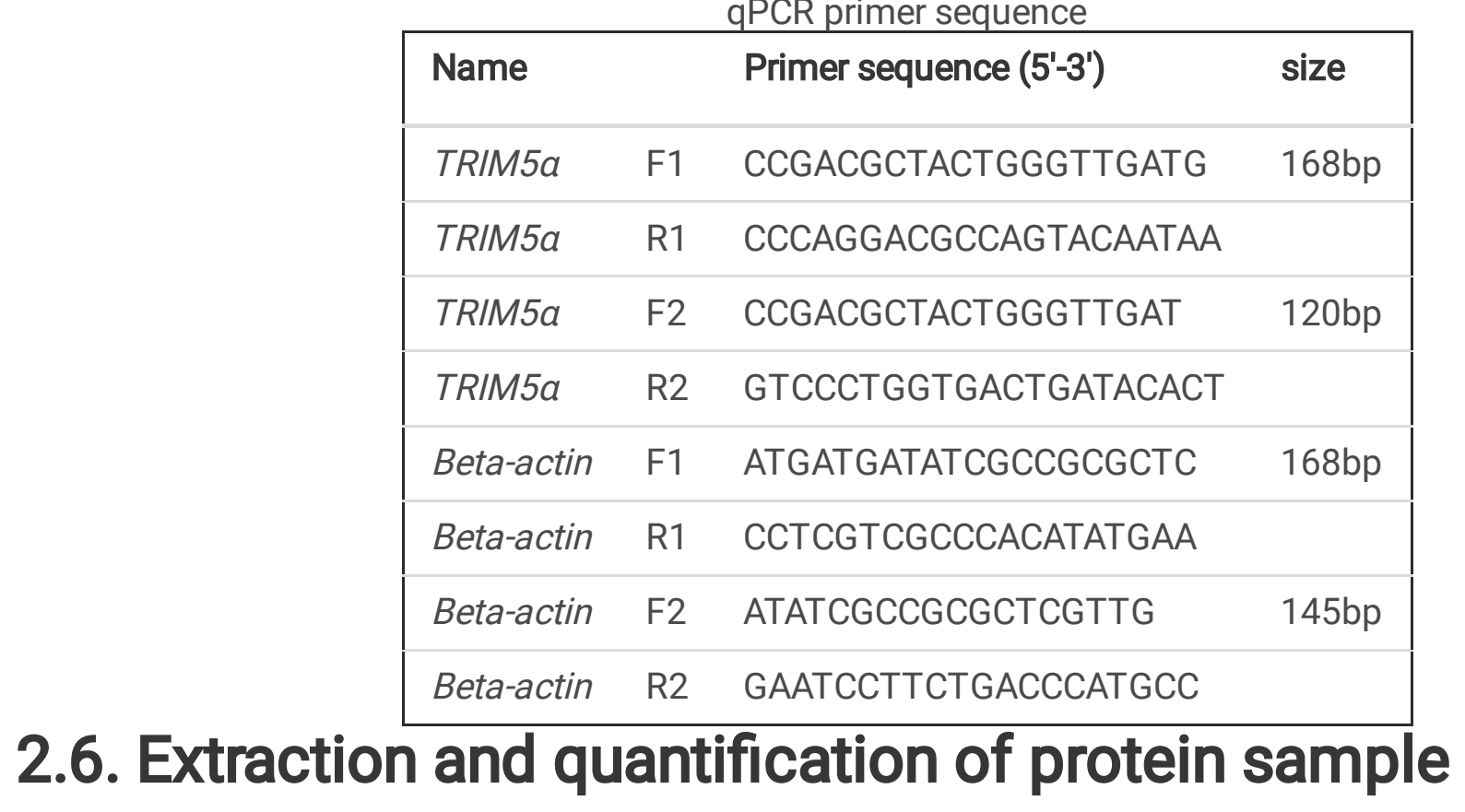

Total proteins were extracted from the snap-frozen CMEF cell pellets using the in lysis buffer solution (50 mM Tris pH 7.5, $150 \mathrm{mM} \mathrm{NaCl}, 10 \%$ glycerol (v/v), 1 mM EDTA, 1 mM EGTA, 1\% NP-40, 1 mM DTT). The lysate was incubated with $100 \mathrm{mM}$ PMSF protease inhibitor (Sigma), centrifuged at $13,000 \mathrm{~g}$ for $1 \mathrm{~min}$ at $4^{\circ} \mathrm{C}$, supernatant containing the cytosolic protein extract collected and stored at $-80^{\circ} \mathrm{C}$ until use. The pellet was re-suspended in extraction buffer (2 $\mathrm{M}$ sucrose, $1 \mathrm{M}$ HEPES, $2 \mathrm{M} \mathrm{MgCl}_{2}, 2 \mathrm{M} \mathrm{KCl}, 30 \%$ glycerol, $0.5 \mathrm{M}$ EDTA, $1 \mathrm{M}$ DTT, protease inhibitor cocktail, and $10 \% \mathrm{NP}-40$ ), centrifuged at $12,000 \mathrm{~g}$ for $15 \mathrm{~min}$ at $4^{\circ} \mathrm{C}$, the supernatant containing the nuclear protein was collected and stored at $-80^{\circ} \mathrm{C}$ for Western blotting.

\subsection{Western blot analysis}

The protein concentration in the CMEF cell extract was determined Protein Assay Kit (Pierce Biotechnology, Rockford, IL, USA) using BCA standard. The total protein $(40 \mathrm{mg})$ from each sample was separated by $12 \%$ SDS-polyacrylamide gel electrophoresis and transferred from the gel onto nitrocellulose membranes (Sigma), followed by immunodetection. For this, the membrane was placed in a blocking solution prepared using Tris- 
buffered saline with $0.1 \%$ Tween- 20 and $5 \%$ non-fat milk and incubated at room temperature for $1 \mathrm{~h}$. The membranes were then incubated overnight with primary antibodies: anti-TRIM5a (Santa Cruz Biotechnology, CA, USA), and endogenous control anti-GAPDH (Santa Cruz Biotechnology, CA, USA) at $4^{\circ} \mathrm{C}$. After the washing step, the membranes were placed in the secondary antibody solution conjugated with horseradish peroxidase (HRP) (Santa Cruz Biotechnology, CA, USA), and the immunoreaction signals were visualized using chemiluminescence detection (Thermo Fisher Scientific, Rockford, IL, USA).

\subsection{Transduction of shTRIM5a lentivirus recombinant vector in HIV Pseudo-virus infected CMEF cells}

The CMEF cells to be transduced were seeded in 48-well plates at a cell density of $1 \times 10^{\wedge} 4$ cells/well and were grown until $70-80 \%$ confluent. In this stage, the cells were transduced with shTRIM5a lentivirus recombinant vector (shRNA1-CMEF, shRNA2-CMEF, shRNA3-CMEF cells) according to the manufacturer's instructions and non-transduced control (NTC-CMEF cells). The TRIM5a gene knockdown in shRNA-CMEF cells was tested by infecting them and control cells with different concentrations $(0.125 \mu \mathrm{L}$ to $4 \mu \mathrm{L})$ of HIV-pseudo-virus load. After $48 \mathrm{~h}$ of incubation, the cells were harvested and tested for GFP and RFP expression.

\section{Results}

The three TRIM5a shRNAs designed and constructed in this study were comparable and matched the target sequence (Fig. 1.B.). The recombinant lentiviral vector containing the target gene shRNA1, shRNA2, and shRNA3 when transfected into the 293T cells showed the genes were successfully packaged into lentivirus vector containing fluorescent protein gene as shown in Fig. 1. C.

The transfected shRNA1-CMEF, shRNA2-CMEF, and shRNA3-CMEF cells stably expressed GFP (Fig. 1.D.). Total mRNA in the samples, including control-CMEF, NTC-CMEF, ShRNA1-CMEF, ShRNA2-CMEF and ShRNA3-CMEF cells, were extracted and the quality were tested (Fig. 2.A.). The expressions of TRIM5a at mRNA level were significantly knockdown compared with the NTC-CMEF cells (Fig. 2.B.). Further, the TRIM5a protein expression levels in wild-type CMEF were found to be $100 \%$ compared to shRNA1-CMEF (59\%), shRNA2-CMEF (47\%) and shRNA3-CMEF (49\%) (Fig. 2.C.), which is consist with western blot analysis (Fig. 2.D.).

Detection of RFP to confirm the knocked-down cells in HIV-pseudo-infected cells showed red fluorescent emissions (Fig. 3.A.).

The levels of RFP emission as a percentage function of GFP expression in ShRNA1-CMEF ShRNA2-CMEF, and ShRNA3-CMEF cells compared to control cells were detected (Fig. 3.B.).

\section{Discussion}

Although there are thousands of protein-encoding genes in the genome of an organism, very less is known about their functions. At the end of the last decade of 20th century, Fire and Craig introduced a novel technique, RNA interference (RNAi), for targeted suppression of individual genes ${ }^{9,13}$. In the RNAi technique, gene sequence anti-sense to the siRNA is knocked down during the post-transcriptional stage. The use of short hairpin RNA (shRNA) in experiments has earlier shown these hairpin structures to be effective than siRNAs ${ }^{14-16}$ as the 
siRNA-based knockdowns depend on both the target sequence and transfection efficiency. The low efficiency of transfection and the longevity of knockdown are the two major limitations of RNAi technology.

Recently, the use of viral vectors such as lentiviruses for the expression of shRNA has been accomplished and has continued to generate great interest for use in gene therapy. Over the past two years, considerable progress has been made in demonstrating the use of shRNA lentiviral delivery to a wide range of tissues, and a wide variety of gene knockdown experiments reflects their relative ease of use. Here for the first time, we have successfully expressed three shRNAs using lentiviral vectors and knocked down TRIM5a expression in CMEF cells. Our results suggested that a stable and long-time expression of shRNAs can be obtained through lentivirus vectors.

Human immunodeficiency virus type 1 (HIV-1) is the causative agent of acquired immunodeficiency syndrome (AIDS) in humans. Different laboratories world over are continually working to develop in vitro mammalian cell models or in vivo animal models against HIV using HIV real or pseudo-virus to infect animal cells. During experimentation, HIV often enters the animal cells efficiently; however, it sometimes encounters block before reverse transcription ${ }^{17-19}$. This species-specific restriction on the incoming HIV-1 capsid is mediated by a dominant repressive factor. There are many different such host cell barrier proteins in the early phase of immunodeficiency virus replication in human and nonhuman primate species such as APOBEC 3G, TRIM5a ${ }^{17,18}$. The inhibitory role rhTRIM5a and cmTRIM5a at an early post-entry stage of HIV-1 infection has been reported earlier $^{20}$. In this study, we have explored the cynomolgus monkey embryonic fibroblast cells as a potential HIV model. The inhibition of HIV-1 infection by shTRIM5a-CMEF knockdown is due to the species-specific mediator response by innate cellular resistance of CMEF cells against pseudo-HIV infection. Additionally, a decrease in the HIV-pseudo virus infection with an increase in virus concentration (Figure 3.B) matches with the previous report stating the ability of huTRIM5a to reduce HIV-1 infection ${ }^{20-22}$. Our research provides an important strategy for the establishment of shRNA-based TRIMa knockdown in the CMEF cell model of HIV infection.

\section{Abbreviations}

TRIM5a

Tripartite motif proteina

CMEFs

Cynomolgus monkey embryonic fibroblast cells

huTRIM5a

Human TRIM5a

N-MLV

$\mathrm{N}$-tropic murine leukemia virus

rmTRIM5a

Rhesus monkey TRIM5a

TRIM5-CypA

TRIM5-Cyclophilin A

SiRNA

Short interfering RNA

SIV 
Simian immunodeficiency virus

VSV G

Vesicular stomatitis virus $\mathrm{G}$

qRT-PCR

Quantitative real-time RT-PCR

HRP

Horseradish peroxidase

\section{Declarations}

\section{Data availability}

All data supporting our findings are included in the manuscript.

\section{Acknowledgements}

Many thanks to Weiqiang Li and Peng Andy Xiang (Stem cell and tissue engineering research center of Sun Yat sen University, China).

\section{Funding}

This work was supported by the Science and technology innovation project of Guangdong Provincial Department of Education『Grant NO: 2013KJCX0029『

\section{Ethics approval and consent to participate}

Not applicable.

\section{Consent for publication}

Not applicable.

\section{Competing interests}

The authors declare no competing interests.

\section{References}

1. Qurban, R., Saeed, S., Kanwal, W., Junaid, K. \& Rehman, A. Potential immune modulatory effect of vitamin D in HIV infection: A review. Clin. Nutr. ESPEN47, 1-8 https://doi.org/10.1016/j.clnesp.2021.12.005 (2022).

2. Weatherley, D. A. V., Boswell, M. T. \& Rowland-Jones, S. L. Targeting TRIM5a in HIV cure strategies for the CRISPR-Cas9 era. Front Immunol. 8, 1616 https://doi.org/10.3389/fimmu.2017.01616 (2017).

3. Stremlau, M. et al. The cytoplasmic body component TRIM5a restricts HIV-1 infection in Old World monkeys. Nature427, 848-853 https://doi.org/10.1038/nature02343 (2004).

4. Perron, M. J. et al. TRIM5a mediates the postentry block to $\mathrm{N}$-tropic murine leukemia viruses in human cells. Proc. Natl. Acad. Sci. U. S. A.101, 11827 LP - 11832 https://doi.org/10.1073/pnas.0403364101 
(2004).

5. Nisole, S., Lynch, C., Stoye, J. P. \& Yap, M. W. A trim5-cyclophilin a fusion protein found in owl monkey kidney cells can restrict HIV-1. Proc. Natl. Acad. Sci. U. S. A.101, 13324 LP - 13328 https://doi.org/10.1073/pnas.0404640101 (2004).

6. Haihan, S. et al. A single amino acid of the human immunodeficiency virus type 2 capsid affects its replication in the presence of cynomolgus monkey and human TRIM5as. J. Virol.81, 7280-7285 https://doi.org/10.1128/JVI.00406-07 (2007).

7. Howe, S. J. et al. Insertional mutagenesis combined with acquired somatic mutations causes leukemogenesis following gene therapy of SCID-X1 patients. J. Clin. Invest.118, 3143-3150 https://doi.org/10.1172/JCl35798 (2008).

8. Marina, C. C. et al. Gene therapy of human severe combined immunodeficiency (SCID)-X1 disease. Science288, 669-672 https://doi.org/10.1126/science.288.5466.669 (2000).

9. Fire, A. et al. Potent and specific genetic interference by double-stranded RNA in Caenorhabditis elegans. Nature391, 806-811 https://doi.org/10.1038/35888 (1998).

10. Krueger, U. et al. Insights into effective RNAi gained from large-scale siRNA validation screening. Oligonucleotides17, 237-250 https://doi.org/10.1089/oli.2006.0065 (2007).

11. Bernards, R., Brummelkamp, T. R. \& Beijersbergen, R. L. shRNA libraries and their use in cancer genetics. Nat. Methods3, 701-706 https://doi.org/10.1038/nmeth921 (2006).

12. Southwick, C. H. \& Siddiqi, M. F. Population status of nonhuman primates in Asia, with emphasis on rhesus macaques in India. Am. J. Primatol.34, 51-59 https://doi.org/10.1002/ajp.1350340110 (1994).

13. Mocellin, S. \& Provenzano, M. RNA interference: learning gene knock-down from cell physiology. J. Transl. Med.2, 39 https://doi.org/10.1186/1479-5876-2-39 (2004).

14. Paddison, P. J., Caudy, A. A., Bernstein, E., Hannon, G. J. \& Conklin, D. S. Short hairpin RNAs (shRNAs) induce sequence-specific silencing in mammalian cells. Genes Dev.16, 948-958 https://doi.org/10.1101/gad.981002 (2002).

15. Thijin R, B., René, B. \& Reuven, A. A System for stable expression of short interfering RNAs in mammalian cells. Science296, 550-553 https://doi.org/10.1126/science.1068999 (2002).

16. McManus, M. T., Petersen, C. P., Haines, B. B., Jianzhu, C. \& Sharp, P. A. Gene silencing using micro-RNA designed hairpins. RNA8, 842-850 https://doi.org/10.1017/S1355838202024032 (2002).

17. Wiegand, H. L., Doehle, B. P., Bogerd, H. P. \& Cullen, B. R. A second human antiretroviral factor, APOBEC3F, is suppressed by the HIV-1 and HIV-2 Vif proteins. EMBO J.23, 2451-2458 https://doi.org/10.1038/sj.emboj.7600246 (2004).

18. Sayah, D. M., Sokolskaja, E., Berthoux, L. \& Luban, J. Cyclophilin A retrotransposition into TRIM5 explains owl monkey resistance to HIV-1. Nature430, 569-573 https://doi.org/10.1038/nature02777 (2004).

19. Sheehy, A. M., Gaddis, N. C., Choi, J. D. \& Malim, M. H. Isolation of a human gene that inhibits HIV-1 infection and is suppressed by the viral Vif protein. Nature418, 646-650 https://doi.org/10.1038/nature00939 (2002).

20. Emi E, N., Hiroyuki, M., Yoshiyuki, N. \& Tatsuo, S. A specific region of 37 amino acid residues in the SPRY (B30.2) domain of african green monkey TRIM5a determines species-specific restriction of simian 
immunodeficiency virus SIVmac infection. J. Virol.79, 8870-8877 https://doi.org/10.1128/JVI.79.14.88708877.2005 (2005).

21. Miyoshi, H., Takahashi, M., Gage, F. H. \& Verma, I. M. Stable and efficient gene transfer into the retina using an HIV-based lentiviral vector. Proc. Natl. Acad. Sci.94, 10319 LP - 10323

https://doi.org/10.1073/pnas.94.19.10319 (1997).

22. Hiroyuki, M., Ulrike, B., Masayo, T., Gage, F. H. \& Verma, I. M. Development of a self-inactivating lentivirus vector. J. Virol.72, 8150-8157 https://doi.org/10.1128/JVI.72.10.8150-8157.1998 (1998).

\section{Figures}
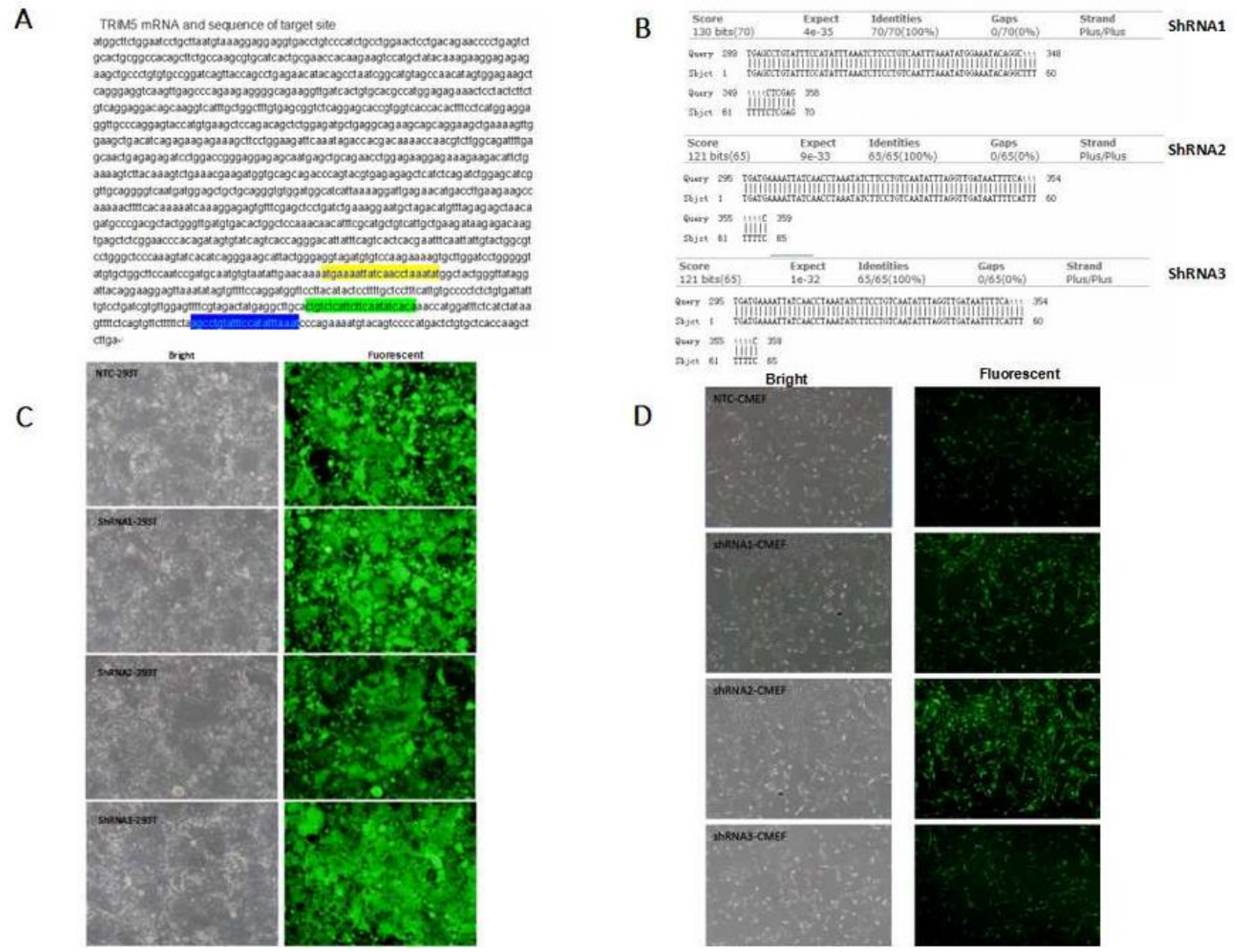

\section{Figure 1}

Screening, synthesis, and validation of shRNA

(a) Cynomolgus monkey TRIM5a mRNA sequence and shRNA knockdown target sites. (b) Comparison of three target plasmid (shRNA1, 2 \& 3) (c) Assessment of transfection of cells with CMEF-shRNA1, $-2,-3$ and Non-target control (NTC) lentivirus vectors (Magnification, x100). (d) Selection of flow-sorted cell lines with TRIM5a knockdown (Magnification, x100). 

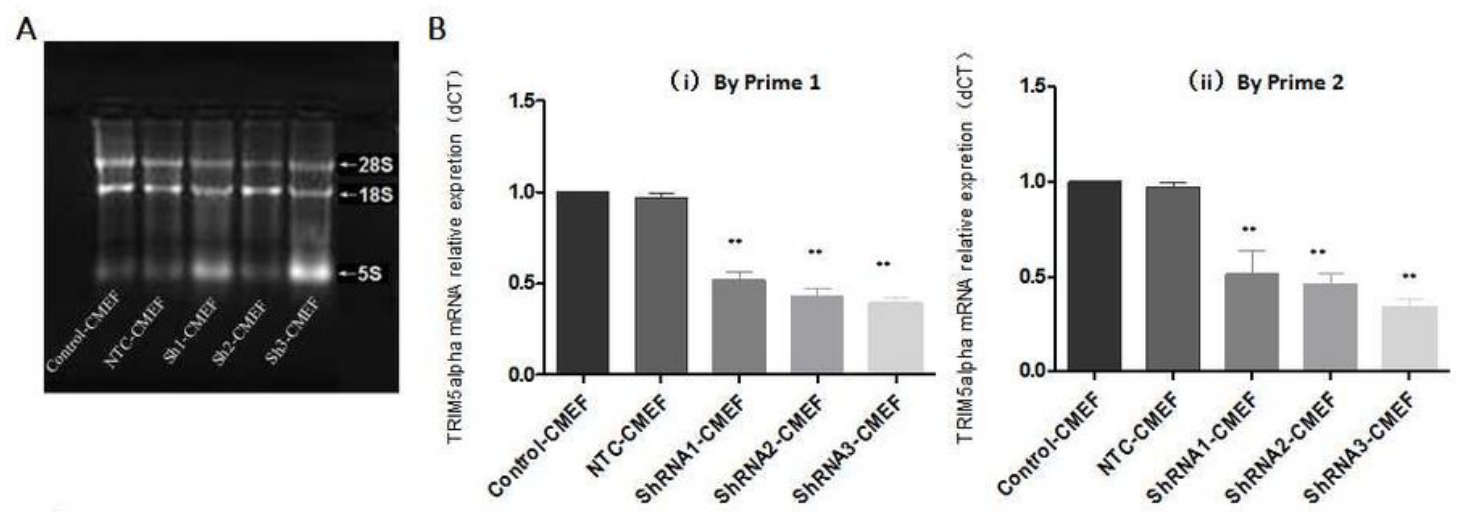

C

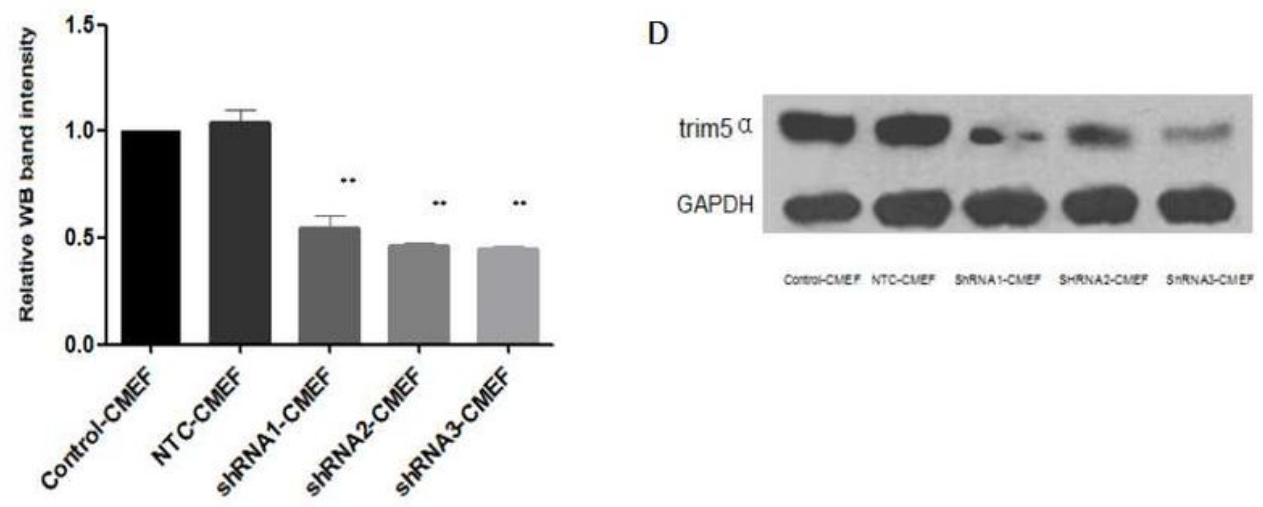

Figure 2

Knockdown efficiency analysis

RNA quality analysis with gel electrophoresis (b) expression of TRIM5a gene in cells by using two different pairs of primers (i) primer pair 1 detect TRIM5a gene expression in 5 cells groups; (ii) Primer 2 to detect TRIM5a gene expression in 5 cells groups. (c) TRIM5A expression was decreased using shRNA against TRIM5A in CMEF cells. (d) Western blot analysis 


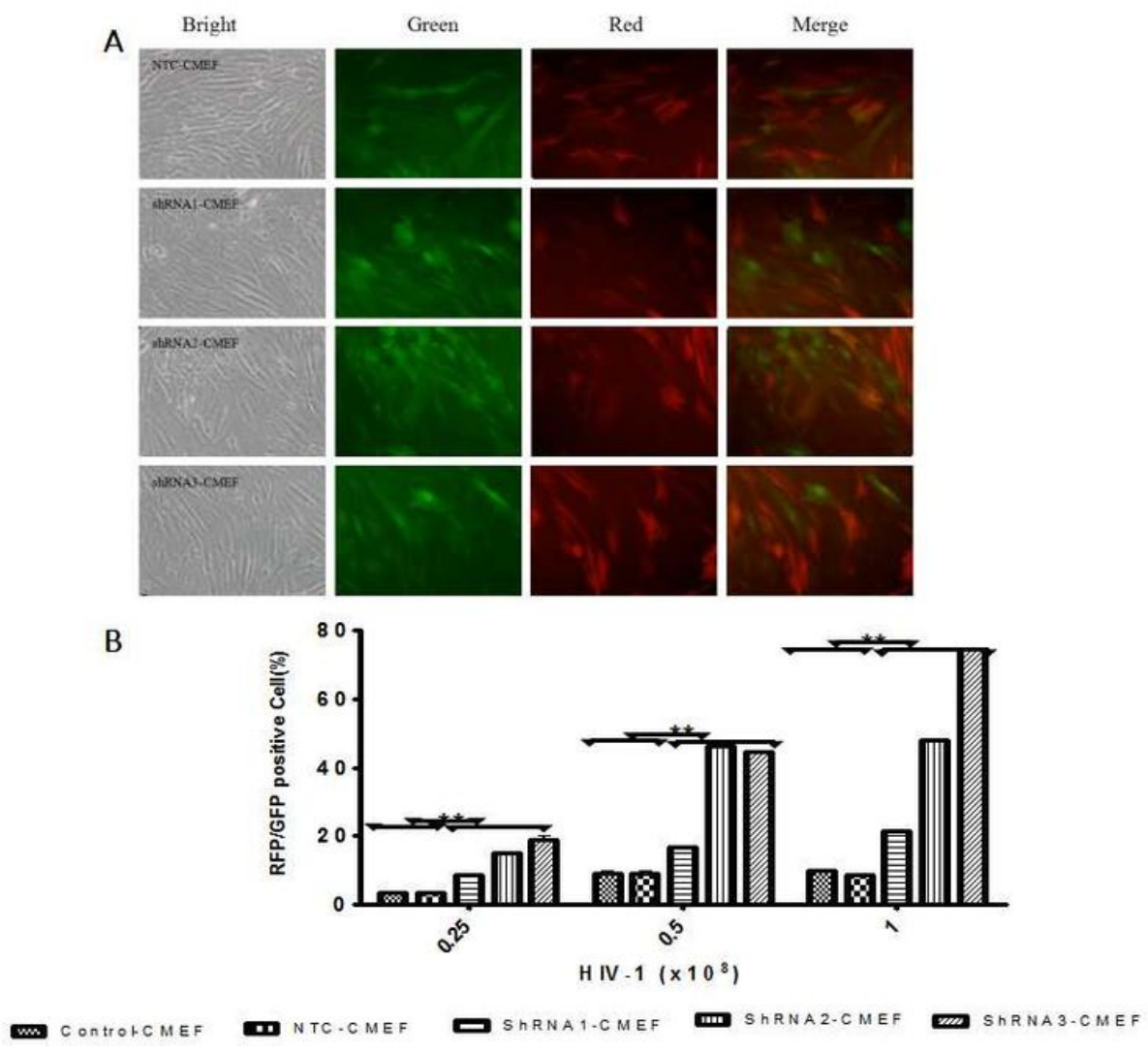

Figure 3

Assessment of GFP-and RFP-expressing cells with the Image-Based Cytometer and Pseudo-HIV infection

(a). (A) NTC-CMEF (B) ShRNA1-CMEF (C) ShRNA2-CMEF (D): ShRNA3-CMEF

(b). Pseudo-HIV infection 